REPRESENTATION THEORY

An Electronic Journal of the American Mathematical Society

Volume 10, Pages 147-157 (March 9, 2006)

S $1088-4165(06) 00285-8$

\title{
AN AVERAGING PROCESS FOR UNIPOTENT GROUP ACTIONS
}

\author{
AMNON YEKUTIELI
}

\begin{abstract}
We present an averaging process for sections of a torsor under a unipotent group. This process allows one to integrate local sections of such a torsor into a global simplicial section. The results of this paper have applications to deformation quantization of algebraic varieties.
\end{abstract}

\section{INTRODUCTION}

Let $\mathbb{K}$ be a field of characteristic 0 . For any natural number $q$ let $\mathbb{K}\left[t_{0}, \ldots, t_{q}\right]$ be the polynomial algebra, and let $\boldsymbol{\Delta}_{\mathbb{K}}^{q}$ be the geometric q-dimensional simplex

$$
\boldsymbol{\Delta}_{\mathbb{K}}^{q}:=\operatorname{Spec} \mathbb{K}\left[t_{0}, \ldots, t_{q}\right] /\left(t_{0}+\cdots+t_{q}-1\right) .
$$

Our main result is the following theorem.

Theorem 0.1. Let $G$ be a unipotent algebraic group over $\mathbb{K}$, let $X$ be a $\mathbb{K}$-scheme, let $Z$ be a $G$-torsor over $X$, and let $Y$ be any $X$-scheme. Suppose $\boldsymbol{f}=\left(f_{0}, \ldots, f_{q}\right)$ is a sequence of $X$-morphisms $f_{i}: Y \rightarrow Z$. Then there is an $X$-morphism

$$
\operatorname{wav}_{G}(\boldsymbol{f}): \boldsymbol{\Delta}_{\mathbb{K}}^{q} \times Y \rightarrow Z
$$

called the weighted average, such that the operation $\operatorname{wav}_{G}$ is symmetric, simplicial, functorial in the data $(G, X, Y, Z)$, and is the identity for $q=0$.

"Symmetric" means that $\operatorname{wav}_{G}$ is equivariant for the action of the permutation group of $\{0, \ldots, q\}$ on the sequence $\boldsymbol{f}$ and the scheme $\boldsymbol{\Delta}_{\mathbb{K}}^{q}$. "Simplicial" says that as $q$ varies

$$
\operatorname{wav}_{G}: \operatorname{Hom}_{X}(Y, Z)^{q+1} \rightarrow \operatorname{Hom}_{X}\left(\boldsymbol{\Delta}_{\mathbb{K}}^{q} \times Y, Z\right)
$$

is a map of simplicial sets. "Functorial in the data $(G, X, Y, Z)$ " means that, e.g., given a morphism $h: Z \rightarrow Z^{\prime}$ of $G$-torsors over $X$, one has

$$
h \circ \operatorname{wav}_{G}(\boldsymbol{f})=\operatorname{wav}_{G}(h \circ \boldsymbol{f}),
$$

where $h \circ f$ is the sequence $\left(h \circ f_{0}, \ldots, h \circ f_{q}\right)$. Theorem 0.1 is repeated, in full detail, as Theorem 1.11

Observe that when we restrict the morphism $\operatorname{wav}_{G}(f)$ to each of the vertices of $\boldsymbol{\Delta}_{\mathbb{K}}^{q}$ we recover the original morphisms $f_{0}, \ldots, f_{q}$. This is due to the simplicial property of $\operatorname{wav}_{G}$. Thus, $\operatorname{wav}_{G}(\boldsymbol{f})$ interpolates between $f_{0}, \ldots, f_{q}$. This is illustrated in Figure 1.

Received by the editors May 11, 2005 and, in revised form, January 3, 2006.

2000 Mathematics Subject Classification. Primary 14L30; Secondary 18G30, 20 G15.

Key words and phrases. Unipotent group, torsor, simplicial set.

This work was partially supported by the US - Israel Binational Science Foundation. 
Here is a naive corollary of Theorem 0.1 , which can further explain the result. Let us write $G(\mathbb{K})$ for the group of $\mathbb{K}$-rational points of $G$. By a weight sequence in $\mathbb{K}$ we mean a sequence $\boldsymbol{w}=\left(w_{0}, \ldots, w_{q}\right)$ of elements of $\mathbb{K}$ such that $\sum w_{i}=1$.

Corollary 0.2. Let $G$ be a unipotent group over $\mathbb{K}$. Suppose $Z$ is a set with $G(\mathbb{K})$-action which is transitive and has trivial stabilizers. Let $\boldsymbol{z}=\left(z_{0}, \ldots, z_{q}\right)$ be a sequence of points in $Z$ and let $\boldsymbol{w}$ be a weight sequence in $\mathbb{K}$. Then there is a point $\operatorname{wav}_{G, \boldsymbol{w}}(\boldsymbol{z}) \in Z$ called the weighted average. The operation $\operatorname{wav}_{G}$ is symmetric, functorial, simplicial, and is the identity for $q=0$.

The corollary is proved in Section 1. The idea is of course to take $Y=X:=$ Spec $\mathbb{K}$ in the theorem, and to note that $\boldsymbol{w}$ is a $\mathbb{K}$-rational point of $\boldsymbol{\Delta}_{\mathbb{K}}^{q}$.

Another consequence of Theorem 0.1 is a new proof of the fact that a unipotent group in characteristic 0 is special. See Remark 1.12. (This observation is due to Reichstein.)

Theorem 0.1 was discovered in the course of research on deformation quantization in algebraic geometry [Ye, in which we tried to apply ideas of Kontsevich [Ko] to the algebraic context. Here is a brief outline. Suppose $X$ is a smooth $n$-dimensional $\mathbb{K}$-scheme. The coordinate bundle of $X \mathbb{G K}, \mathrm{Ko}$ is an infinite dimensional bundle $Z \rightarrow X$ which parameterizes formal coordinate systems on $X$. The bundle $Z$ is a torsor under an affine group scheme $\mathrm{GL}_{n, \mathbb{K}} \ltimes G$, where $G$ is pro-unipotent. One is interested in sections of the quotient bundle $\bar{Z}:=Z / \mathrm{GL}_{n, \mathbb{K}}$. If we are in the differentiable setup (i.e. $\mathbb{K}=\mathbb{R}$ and $X$ is a $\mathrm{C}^{\infty}$ manifold), then the fibers of $\bar{Z}$ are contractible (since they are isomorphic to $G(\mathbb{R})$ ), and therefore global $\mathrm{C}^{\infty}$ sections exist. However, in the setup of algebraic geometry such an argument is invalid, and we were forced to seek an alternative approach.

Our solution was to use simplicial sections of $\bar{Z}$ (see Section 2, and especially Corollary 2.7]). This approach was inspired by work of Bott Bo. on simplicial connections (cf. also [HY]).

Acknowledgments. The author thanks David Kazhdan and Zinovy Reichstein for useful conversations. Also, thanks to the referee for reading the paper carefully and suggesting a few improvements.

\section{The Averaging Process}

Throughout the paper $\mathbb{K}$ denotes a fixed base field of characteristic 0 . All schemes and all morphisms are over $\mathbb{K}$.

We begin by recalling some standard facts about the combinatorics of simplicial objects. Let $\boldsymbol{\Delta}$ denote the category with objects the ordered sets $[q]:=\{0,1, \ldots, q\}$, $q \in \mathbb{N}$. The morphisms $[p] \rightarrow[q]$ are the order-preserving functions, and we write $\boldsymbol{\Delta}_{p}^{q}:=\operatorname{Hom}_{\boldsymbol{\Delta}}([p],[q])$. The $i$-th co-face map $\partial^{i}:[p] \rightarrow[p+1]$ is the injective function that does not take the value $i$; and the $i$-th co-degeneracy map $\mathrm{s}^{i}:[p] \rightarrow[p-1]$ is the surjective function that takes the value $i$ twice. All morphisms in $\boldsymbol{\Delta}$ are compositions of various $\partial^{i}$ and $\mathrm{s}^{i}$.

An element of $\boldsymbol{\Delta}_{p}^{q}$ may be thought of as a sequence $\boldsymbol{i}=\left(i_{0}, \ldots, i_{p}\right)$ of integers with $0 \leq i_{0} \leq \cdots \leq i_{p} \leq q$. Given $\boldsymbol{i} \in \boldsymbol{\Delta}_{q}^{m}, \boldsymbol{j} \in \boldsymbol{\Delta}_{m}^{p}$ and $\alpha \in \boldsymbol{\Delta}_{p}^{q}$, we sometimes write $\alpha_{*}(\boldsymbol{i}):=\boldsymbol{i} \circ \alpha \in \boldsymbol{\Delta}_{p}^{m}$ and $\alpha^{*}(\boldsymbol{j}):=\alpha \circ \boldsymbol{j} \in \boldsymbol{\Delta}_{m}^{q}$.

Let $\mathrm{C}$ be some category. A cosimplicial object in $\mathrm{C}$ is a functor $C: \boldsymbol{\Delta} \rightarrow \mathrm{C}$. We shall usually refer to the cosimplicial object as $C=\left\{C^{p}\right\}_{p \in \mathbb{N}}$, and for any $\alpha \in \boldsymbol{\Delta}_{p}^{q}$ the corresponding morphism in $\mathrm{C}$ will be denoted by $\alpha^{*}: C^{p} \rightarrow C^{q}$. A simplicial 
object in $C$ is a functor $C: \Delta^{\mathrm{op}} \rightarrow \mathrm{C}$. The notation for a simplicial object will be $C=\left\{C_{p}\right\}_{p \in \mathbb{N}}$ and $\alpha_{*}: C_{q} \rightarrow C_{p}$.

An important example is the cosimplicial scheme $\left\{\boldsymbol{\Delta}_{\mathbb{K}}^{p}\right\}_{p \in \mathbb{N}}$. The morphisms are defined as follows. For any $p$ we identify the ordered set $[p]$ with the set of vertices of $\boldsymbol{\Delta}_{\mathbb{K}}^{p}$. Given $\alpha \in \boldsymbol{\Delta}_{p}^{q}$, the morphism $\alpha^{*}: \boldsymbol{\Delta}_{\mathbb{K}}^{p} \rightarrow \boldsymbol{\Delta}_{\mathbb{K}}^{q}$ is then the unique linear morphism extending $\alpha:[p] \rightarrow[q]$.

Let $G$ be a unipotent (affine finite type) algebraic group over $\mathbb{K}$, with (nilpotent) Lie algebra $\mathfrak{g}$. We write $d(G)$ for the minimal number $d$ such that there exists a chain of closed normal subgroups $G=G_{0} \supset G_{1} \cdots \supset G_{d}=1$ with $G_{k} / G_{k+1}$ abelian for all $k \in\{0, \ldots, d-1\}$. The exponential map $\exp _{G}: \mathfrak{g} \rightarrow G$ is an isomorphism of schemes, with inverse $\log _{G}$; see [Ho, Theorem VIII.1.1].

Given a $\mathbb{K}$-scheme $X$, there is a Lie algebra $\mathfrak{g} \times X$ (in the category of $X$-schemes), and a group-scheme $G \times X$. There is also an induced exponential map

$$
\exp _{G \times X}:=\exp _{G} \times \mathbf{1}_{X}: \mathfrak{g} \times X \rightarrow G \times X .
$$

We will need the following result.

Lemma 1.1. Let $G, G^{\prime}$ be two unipotent groups, with Lie algebras $\mathfrak{g}, \mathfrak{g}^{\prime}$. Let $X, X^{\prime}$ be schemes, let $X \rightarrow X^{\prime}$ be a morphism of schemes, and let $\phi: G \times X \rightarrow G^{\prime} \times X^{\prime}$ be a morphism of group-schemes over $X^{\prime}$. Denote by $\mathrm{d} \phi: \mathfrak{g} \times X \rightarrow \mathfrak{g}^{\prime} \times X^{\prime}$ the induced Lie algebra morphism (the differential of $\phi$ ). Then the diagram

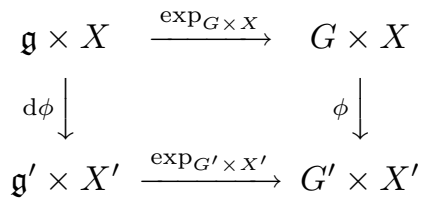

commutes.

Proof. For the case $X=X^{\prime}=$ Spec $\mathbb{K}$ this is contained in the proof of $\mathrm{Ho}$, Theorem VIII.1.2].

In order to handle the general case we first recall the Campbell-Baker-Hausdorff formula:

$$
\exp _{G}\left(\gamma_{1}\right) \cdot \exp _{G}\left(\gamma_{2}\right)=\exp _{G}\left(F\left(\gamma_{1}, \gamma_{2}\right)\right)
$$

where

$$
F\left(\gamma_{1}, \gamma_{2}\right)=\gamma_{1}+\gamma_{2}+\frac{1}{2}\left[\gamma_{1}, \gamma_{2}\right]+\cdots
$$

is a universal power series (see [Ho, Section XVI.2]). Hence if we define $\gamma_{1} * \gamma_{2}:=$ $F\left(\gamma_{1}, \gamma_{2}\right)$, then $(\mathfrak{g}, *)$ becomes an algebraic group (with unit element 0 ), and $\exp _{G}$ : $(\mathfrak{g}, *) \rightarrow(G, \cdot)$ is a group isomorphism. In this way we may eliminate $G$ altogether, and just look at the scheme $\mathfrak{g}$ with its two structures: a Lie algebra and a groupscheme. Note that now $\mathfrak{g}$ is its own Lie algebra, as can be seen from the second-order term in the series $F\left(\gamma_{1}, \gamma_{2}\right)$.

Consider a morphism $\phi: \mathfrak{g} \times X \rightarrow \mathfrak{g}^{\prime} \times X^{\prime}$ of $X^{\prime}$-schemes. Then $\phi$ is a morphism of Lie algebras over $X^{\prime}$ iff it is a morphism of group-schemes (for the multiplications $*$ ) and, moreover, $\mathrm{d} \phi=\phi$. Therefore, the diagram (1.2) is commutative.

From now on we will write $\exp _{G}$ instead of $\exp _{G \times X}$, for the sake of brevity.

Consider the following setup: $X$ is a $\mathbb{K}$-scheme, and $Y, Z$ are two $X$-schemes. Suppose $Z$ is a torsor under the group scheme $G \times X$. We denote the action of $G$ on $Z$ by $(g, z) \mapsto g \cdot z$. 
Let $f_{0}, \ldots, f_{q}: \boldsymbol{\Delta}_{\mathbb{K}}^{q} \times Y \rightarrow Z$ be $X$-morphisms. We are going to define a new sequence of $X$-morphisms $f_{0}^{\prime}, \ldots, f_{q}^{\prime}: \boldsymbol{\Delta}_{\mathbb{K}}^{q} \times Y \rightarrow Z$. Because $Z$ is a torsor under $G \times X$, for any $i, j \in\{0, \ldots, q\}$ there exists a unique morphism $g_{i, j}: \boldsymbol{\Delta}_{\mathbb{K}}^{q} \times Y \rightarrow G$ such that $f_{j}(w, y)=g_{i, j}(w, y) \cdot f_{i}(w, y)$ for all points $w \in \boldsymbol{\Delta}_{\mathbb{K}}^{q}$ and $y \in Y$. Here $w$ and $y$ are scheme-theoretic points, i.e., $w \in \boldsymbol{\Delta}_{\mathbb{K}}^{q}(U)=\operatorname{Hom}_{\mathbb{K}}\left(U, \boldsymbol{\Delta}_{\mathbb{K}}^{q}\right)$ and $y \in$ $Y(U)=\operatorname{Hom}_{\mathbb{K}}(U, Y)$ for some $\mathbb{K}$-scheme $U$. Define

$$
f_{i}^{\prime}(w, y):=\exp _{G}\left(\sum_{j=0}^{q} t_{j}(w) \cdot \log _{G}\left(g_{i, j}(w, y)\right)\right) \cdot f_{i}(w, y) .
$$

In this formula we view $t_{j}$ as a morphism $t_{j}: \boldsymbol{\Delta}_{\mathbb{K}}^{q} \rightarrow \mathbf{A}_{\mathbb{K}}^{1}$, and we use the fact that $\mathfrak{g}$ is a vector space (in the category of $\mathbb{K}$-schemes).

For any set $S$ let us write $S^{\Delta_{0}^{q}}$ for the set of functions $\Delta_{0}^{q} \rightarrow S$, which is the same as the set of sequences $\left(s_{0}, \ldots, s_{q}\right)$ in $S$. As usual $\operatorname{Hom}_{X}\left(\boldsymbol{\Delta}_{\mathbb{K}}^{q} \times Y, Z\right)$ is the set of $X$-morphisms $\boldsymbol{\Delta}_{\mathbb{K}}^{q} \times Y \rightarrow Z$. In this notation the sequences $\left(f_{0}, \ldots, f_{q}\right)$ and $\left(f_{0}^{\prime}, \ldots, f_{q}^{\prime}\right)$ are elements of $\operatorname{Hom}_{X}\left(\boldsymbol{\Delta}_{\mathbb{K}}^{q} \times Y, Z\right)^{\boldsymbol{\Delta}_{0}^{q}}$. We denote by

$$
\operatorname{wsym}_{G}: \operatorname{Hom}_{X}\left(\boldsymbol{\Delta}_{\mathbb{K}}^{q} \times Y, Z\right)^{\boldsymbol{\Delta}_{0}^{q}} \rightarrow \operatorname{Hom}_{X}\left(\boldsymbol{\Delta}_{\mathbb{K}}^{q} \times Y, Z\right)^{\boldsymbol{\Delta}_{0}^{q}}
$$

the operation $\left(f_{0}, \ldots, f_{q}\right) \mapsto\left(f_{0}^{\prime}, \ldots, f_{q}^{\prime}\right)$ given by the formula (1.3).

We will also need a similar operation

$$
\mathrm{w}_{G}: \operatorname{Hom}_{X}(Y, Z)^{\boldsymbol{\Delta}_{0}^{q}} \rightarrow \operatorname{Hom}_{X}\left(\boldsymbol{\Delta}_{\mathbb{K}}^{q} \times Y, Z\right)^{\boldsymbol{\Delta}_{0}^{q}},
$$

defined by

$$
\mathrm{w}_{G}\left(f_{0}, \ldots, f_{q}\right):=\left(f_{0}^{\prime}, \ldots, f_{q}^{\prime}\right)
$$

with

$$
f_{i}^{\prime}(w, y):=\exp _{G}\left(\sum_{j=0}^{q} t_{j}(w) \cdot \log _{G}\left(g_{i, j}(y)\right)\right) \cdot f_{i}(y) .
$$

It is clear that for $q=0$ both operations $\mathrm{w}_{G}$ and wsym $_{G}$ act as the identity, i.e., $\mathrm{w}_{G}\left(f_{0}\right)=\operatorname{wsym}_{G}\left(f_{0}\right)=f_{0}$ for all $f_{0} \in \operatorname{Hom}_{X}(Y, Z)$. Both operations $\mathrm{w}_{G}$ and wsym $_{G}$ are symmetric, namely they are equivariant for the simultaneous action of the permutation group of $\{0, \ldots, q\}$ on $\boldsymbol{\Delta}_{0}^{q}$ and $\boldsymbol{\Delta}_{\mathbb{K}}^{q}$. Also, if $\boldsymbol{f}=\left(f_{0}, \ldots, f_{q}\right) \in$ $\operatorname{Hom}_{X}\left(\boldsymbol{\Delta}_{\mathbb{K}}^{q} \times Y, Z\right)^{\boldsymbol{\Delta}_{0}^{q}}$ is a constant sequence, i.e., $f_{0}=\cdots=f_{q}$, then $\operatorname{wsym}_{G}(\boldsymbol{f})=$ $f$.

Lemma 1.5. Both operations $\mathrm{w}_{G}$ and $\mathrm{wsym}_{G}$ are simplicial. Namely, given $\alpha \in$ $\Delta_{p}^{q}$ the diagram

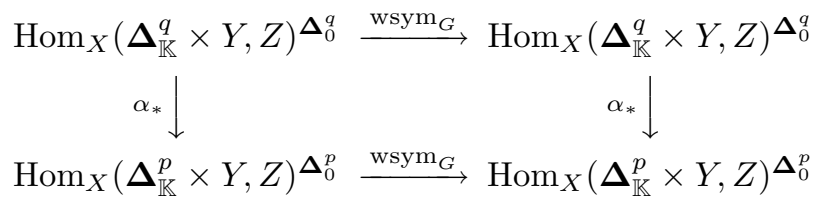

is commutative, and likewise for $\mathrm{w}_{G}$.

Proof. It suffices to consider $\alpha=\partial^{i}$ or $\alpha=\mathrm{s}^{i}$. Since $\operatorname{wsym}_{G}$ is symmetric, we may assume that $\alpha=\partial^{q}:[q-1] \rightarrow[q]$ or $\alpha=\mathrm{s}^{q}:[q+1] \rightarrow[q]$. Fix a sequence $\boldsymbol{f}=\left(f_{0}, \ldots, f_{q}\right) \in \operatorname{Hom}_{X}\left(\boldsymbol{\Delta}_{\mathbb{K}}^{q} \times Y, Z\right)^{\boldsymbol{\Delta}_{0}^{q}}$. Let $g_{i, j} \in \operatorname{Hom}_{\mathbb{K}}\left(\boldsymbol{\Delta}_{\mathbb{K}}^{q} \times Y, G\right)$ be such that $f_{j}=g_{i, j} \cdot f_{i}$, and let $\boldsymbol{f}^{\prime}=\left(f_{0}^{\prime}, \ldots, f_{q}^{\prime}\right):=\operatorname{wsym}_{G}(\boldsymbol{f})$. 
First we look at the case $\alpha=\partial^{q}$. Take $w \in \Delta_{\mathbb{K}}^{q-1}$ and $y \in Y$, and let $v:=$ $\alpha^{*}(w) \in \boldsymbol{\Delta}_{\mathbb{K}}^{q}$. The coordinates of $v$ are $t_{j}(v)=t_{j}(w)$ for $j \leq q-1$, and $t_{q}(v)=0$. Then for any $i$ the $i$-th term in the sequence $\alpha_{*}\left(\boldsymbol{f}^{\prime}\right)$, evaluated at $(w, y)$, equals

$$
\begin{aligned}
f_{i}^{\prime}(v, y) & =\exp _{G}\left(\sum_{j=0}^{q} t_{j}(v) \cdot \log _{G}\left(g_{i, j}(v, y)\right)\right) \cdot f_{i}(v, y) \\
& =\exp _{G}\left(\sum_{j=0}^{q-1} t_{j}(w) \cdot \log _{G}\left(g_{i, j}(v, y)\right)\right) \cdot f_{i}(v, y) .
\end{aligned}
$$

On the other hand, the $i$-th term of the sequence $\operatorname{wsym}_{G}\left(\alpha_{*}(\boldsymbol{f})\right)$ is

$$
\begin{gathered}
\exp _{G}\left(\sum_{j=0}^{q-1} t_{j}(w) \cdot \log _{G}\left(\alpha_{*}\left(g_{i, j}\right)(w, y)\right)\right) \cdot \alpha_{*}\left(f_{i}\right)(w, y) \\
=\exp _{G}\left(\sum_{j=0}^{q-1} t_{j}(w) \cdot \log _{G}\left(g_{i, j}(v, y)\right)\right) \cdot f_{i}(v, y) .
\end{gathered}
$$

So, indeed, $\alpha_{*} \circ$ wsym $_{G}=\operatorname{wsym}_{G} \circ \alpha_{*}$ in this case.

Next consider the case $\alpha=\mathrm{s}^{q}$. Take $w \in \Delta_{\mathbb{K}}^{q+1}$ and $y \in Y$, and let $v:=$ $\alpha^{*}(w) \in \boldsymbol{\Delta}_{\mathbb{K}}^{q}$. The coordinates of $v$ are $t_{j}(v)=t_{j}(w)$ for $j \leq q-1$, and $t_{q}(v)=$ $t_{q}(w)+t_{q+1}(w)$. For any $i \leq q$ the $i$-th term in the sequence $\alpha_{*}(\boldsymbol{f})$, evaluated at $(w, y)$, is $f_{i}^{\prime}(v, y)$, which was calculated in (1.6). The $(q+1)$-st term is also $f_{q}^{\prime}(v, y)$. On the other hand, for any $i \leq q$ the $i$-th term in the sequence $\operatorname{wsym}_{G}\left(\alpha_{*}\left(\boldsymbol{f}^{\prime}\right)\right)$, evaluated at $(w, y)$, is

$$
z_{i}:=\exp _{G}\left(\sum_{j=0}^{q+1} t_{j}(w) \cdot \log _{G}\left(\alpha_{*}\left(g_{i, j}\right)(w, y)\right)\right) \cdot \alpha_{*}\left(f_{i}\right)(w, y) .
$$

But $t_{q}(w)+t_{q+1}(w)=t_{q}(v), \alpha_{*}\left(g_{i, j}\right)(w, y)=g_{i, j}(v, y)$ for $j \leq q$, and $\alpha_{*}\left(g_{i, q+1}\right)(w, y)=g_{i, q}(v, y)$. Therefore,

$$
z_{i}=\exp _{G}\left(\sum_{j=0}^{q} t_{j}(v) \cdot \log _{G}\left(g_{i, j}(v, y)\right)\right) \cdot f_{i}(v, y)
$$

For $i=q+1$ one has $z_{q+1}=z_{q}$. We conclude that $\alpha_{*} \circ \operatorname{wsym}_{G}=\operatorname{wsym}_{G} \circ \alpha_{*}$ in this case too.

The proof for $\mathrm{w}_{G}$ is the same.

Lemma 1.7. Both operations $\mathrm{w}_{G}$ and $\mathrm{wsym}_{G}$ are functorial in the data $(G, X, Y, Z)$. Namely, given another such quadruple $\left(G^{\prime}, X^{\prime}, Y^{\prime}, Z^{\prime}\right)$, a morphism of schemes $X \rightarrow X^{\prime}$, a morphism of schemes $e: Y^{\prime} \rightarrow Y$ over $X^{\prime}$, a morphism of group-schemes $\phi: G \times X \rightarrow G^{\prime} \times X^{\prime}$ over $X^{\prime}$, and a $G \times X$-equivariant morphism of schemes $f: Z \rightarrow Z^{\prime}$ over $X^{\prime}$, the diagram

$$
\begin{array}{cc}
\operatorname{Hom}_{X}\left(\boldsymbol{\Delta}_{\mathbb{K}}^{q} \times Y, Z\right)^{\boldsymbol{\Delta}_{0}^{q}} \stackrel{\operatorname{wsym}_{G}}{\longrightarrow} & \operatorname{Hom}_{X}\left(\boldsymbol{\Delta}_{\mathbb{K}}^{q} \times Y, Z\right)^{\boldsymbol{\Delta}_{0}^{q}} \\
(e, f) \downarrow & (e, f) \downarrow \\
\operatorname{Hom}_{X^{\prime}}\left(\boldsymbol{\Delta}_{\mathbb{K}}^{q} \times Y^{\prime}, Z^{\prime}\right)^{\boldsymbol{\Delta}_{0}^{q}} \stackrel{\mathrm{wsym}_{G^{\prime}}}{\longrightarrow} \operatorname{Hom}_{X^{\prime}}\left(\boldsymbol{\Delta}_{\mathbb{K}}^{q} \times Y^{\prime}, Z^{\prime}\right)^{\boldsymbol{\Delta}_{0}^{q}}
\end{array}
$$

is commutative, and likewise for $\mathrm{w}_{G}$.

Proof. This is due to the functoriality of the exponential map; see Lemma 1.1. 
Lemma 1.8. Assume $G$ is abelian. Then for any $\left(f_{0}, \ldots, f_{q}\right) \in \operatorname{Hom}_{X}\left(\boldsymbol{\Delta}_{\mathbb{K}}^{q} \times\right.$ $Y, Z)^{\Delta_{0}^{q}}$ the sequence $\operatorname{wsym}_{G}\left(f_{0}, \ldots, f_{q}\right)$ is constant.

Proof. In this case exp : $\mathfrak{g} \rightarrow G$ is an isomorphism of algebraic groups, where $\mathfrak{g}$ is viewed as an additive group. So we may assume that $Z$ is a torsor under $\mathfrak{g} \times X$. Let $\left(f_{0}^{\prime}, \ldots, f_{q}^{\prime}\right):=\operatorname{wsym}_{G}\left(f_{0}, \ldots, f_{q}\right)$, and let $\gamma_{i, j}: \boldsymbol{\Delta}_{\mathbb{K}}^{q} \times Y \rightarrow \mathfrak{g}$ be morphisms such that $f_{j}=\gamma_{i, j}+f_{i}$. Take $(w, y) \in \boldsymbol{\Delta}_{\mathbb{K}}^{q} \times Y$. Then

$$
f_{i}^{\prime}(w, y)=\left(\sum_{j=0}^{q} t_{j}(w) \cdot \gamma_{i, j}(w, y)\right)+f_{i}(w, y)
$$

for any $i$. Because $\gamma_{i, j}=-\gamma_{j, i}=\gamma_{0, j}-\gamma_{0, i}, f_{i}=f_{0}+\gamma_{0, i}$ and $\sum_{j=0}^{q} t_{j}(w)=1$, it follows that $f_{i}^{\prime}(w, y)=f_{0}^{\prime}(w, y)$.

We write $\operatorname{wsym}_{G}^{d}$ for the $d$-th iteration of the operation $\operatorname{wsym}_{G}$.

Lemma 1.9. For any $\boldsymbol{f}=\left(f_{0}, \ldots, f_{q}\right) \in \operatorname{Hom}_{X}\left(\boldsymbol{\Delta}_{\mathbb{K}}^{q} \times Y, Z\right)^{\boldsymbol{\Delta}_{0}^{q}}$, the sequence $\operatorname{wsym}_{G}^{d(G)}(\boldsymbol{f})$ is constant. For any $d \geq d(G)$, one has $\operatorname{wsym}_{G}^{d}(\boldsymbol{f})=\operatorname{wsym}_{G}^{d(G)}(\boldsymbol{f})$.

Proof. For any $k$, the orbit of $f_{0} \in \operatorname{Hom}_{X}\left(\boldsymbol{\Delta}_{\mathbb{K}}^{q} \times Y, Z\right)$ under the action of the group $G_{k}\left(\boldsymbol{\Delta}_{\mathbb{K}}^{q} \times Y\right)$ will be denoted by $G_{k}\left(\boldsymbol{\Delta}_{\mathbb{K}}^{q} \times Y\right) \cdot f_{0}$. Let $\left(f_{0}^{\prime}, \ldots, f_{q}^{\prime}\right):=$ $\operatorname{wsym}_{G}\left(f_{0}, \ldots, f_{q}\right)$. We will prove that if $f_{1}, \ldots, f_{q} \in G_{k}\left(\boldsymbol{\Delta}_{\mathbb{K}}^{q} \times Y\right) \cdot f_{0}$, then $f_{1}^{\prime}, \ldots, f_{q}^{\prime} \in G_{k+1}\left(\boldsymbol{\Delta}_{\mathbb{K}}^{q} \times Y\right) \cdot f_{0}^{\prime}$. The assertions of the lemma will then follow.

Let $\tilde{Y}=\tilde{X}:=\boldsymbol{\Delta}_{\mathbb{K}}^{q} \times Y$ and $\tilde{Z}:=\tilde{X} \times_{X} Z$. So $\tilde{Z}$ is a torsor under $G \times \tilde{X}$, and $f_{0}$ induces a morphism $\tilde{f}_{0} \in \operatorname{Hom}_{\tilde{X}}(\tilde{Y}, \tilde{Z})$. The morphism $\tau: G \times \tilde{X} \rightarrow \tilde{Z}$, $(g, \tilde{x}) \mapsto g \cdot \tilde{f}_{0}(\tilde{x})$, is an isomorphism of $\tilde{X}$-schemes. Define $\tilde{W}:=\tau\left(G_{k} \times \tilde{X}\right) \subset \tilde{Z}$. Then $\tilde{W}$ is the "geometric orbit" of $\tilde{f}_{0}$ under $G_{k} \times \tilde{X}$; and, in particular, $\tilde{W}$ is a torsor under $G_{k} \times \tilde{X}$. By assumption $\tilde{f}_{1}, \ldots, \tilde{f}_{q} \in \operatorname{Hom}_{\tilde{X}}(\tilde{Y}, \tilde{W})$. Define $\left(\tilde{f}_{0}^{\prime}, \ldots, \tilde{f}_{q}^{\prime}\right):=$ $\operatorname{wsym}_{G_{k}}\left(\tilde{f}_{0}, \ldots, \tilde{f}_{q}\right)$. By Lemma 1.7 it suffices to prove that $\tilde{f}_{1}^{\prime}, \ldots, \tilde{f}_{q}^{\prime} \in G_{k+1}(\tilde{Y})$. $\tilde{f}_{0}^{\prime}$.

Define $\bar{W}:=\tilde{W} / G_{k+1}$. This is a torsor under the group scheme $\left(G_{k} / G_{k+1}\right) \times \tilde{X}$. Let $\bar{f}_{0}, \ldots, \bar{f}_{q} \in \operatorname{Hom}_{\tilde{X}}(\tilde{Y}, \bar{W})$ be the images of $\left(\tilde{f}_{0}, \ldots, \tilde{f}_{q}\right)$. Because the group $G_{k} / G_{k+1}$ is abelian, Lemma 1.8 says that $\operatorname{wsym}_{G_{k} / G_{k+1}}\left(\bar{f}_{0}, \ldots, \bar{f}_{q}\right)$ is a constant sequence. Again using Lemma1.7 we see that, in fact, $\tilde{f}_{1}^{\prime}, \ldots, \tilde{f}_{q}^{\prime} \in G_{k+1}(\tilde{Y}) \cdot \tilde{f}_{0}^{\prime}$.

Given an $X$-scheme $Y$, the collections $\left\{\operatorname{Hom}_{X}\left(\boldsymbol{\Delta}_{\mathbb{K}}^{q} \times Y, Z\right)\right\}_{q \in \mathbb{N}}$ and $\left\{\operatorname{Hom}_{X}(Y, Z)^{\boldsymbol{\Delta}_{0}^{q}}\right\}_{q \in \mathbb{N}}$ are simplicial sets. For $q=0$ there are equalities

$$
\operatorname{Hom}_{X}\left(\boldsymbol{\Delta}_{\mathbb{K}}^{0} \times Y, Z\right)=\operatorname{Hom}_{X}(Y, Z)=\operatorname{Hom}_{X}(Y, Z)^{\boldsymbol{\Delta}_{0}^{0}} .
$$

Theorem 1.11. Let $G$ be a unipotent algebraic group over $\mathbb{K}$, let $X$ be a $\mathbb{K}$-scheme, and let $Z \rightarrow X$ be a $G$-torsor over $X$. For any $X$-scheme $Y$ and natural number $q$ there is a function

$$
\operatorname{wav}_{G}: \operatorname{Hom}_{X}(Y, Z)^{\boldsymbol{\Delta}_{0}^{q}} \rightarrow \operatorname{Hom}_{X}\left(\boldsymbol{\Delta}_{\mathbb{K}}^{q} \times Y, Z\right)
$$

called the weighted average. The function $\operatorname{wav}_{G}$ enjoys the following properties.

(1) Symmetric: $\operatorname{wav}_{G}$ is equivariant for the action of the permutation group of $\{0, \ldots, q\}$ on $\boldsymbol{\Delta}_{0}^{q}$ and on $\boldsymbol{\Delta}_{\mathbb{K}}^{q}$.

(2) Simplicial: $\operatorname{wav}_{G}$ is a map of simplicial sets

$$
\left\{\operatorname{Hom}_{X}(Y, Z)^{\boldsymbol{\Delta}_{0}^{q}}\right\}_{q \in \mathbb{N}} \rightarrow\left\{\operatorname{Hom}_{X}\left(\boldsymbol{\Delta}_{\mathbb{K}}^{q} \times Y, Z\right)\right\}_{q \in \mathbb{N}} .
$$


(3) Functorial: given another such quadruple $\left(G^{\prime}, X^{\prime}, Y^{\prime}, Z^{\prime}\right)$, a morphism of schemes $X \rightarrow X^{\prime}$, a morphism of $X^{\prime}$-group-schemes $G \times X \rightarrow G^{\prime} \times X^{\prime}$, a $G \times X$-equivariant morphism of $X^{\prime}$-schemes $f: Z \rightarrow Z^{\prime}$ and a morphism of $X^{\prime}$-schemes e: $Y^{\prime} \rightarrow Y$, the diagram

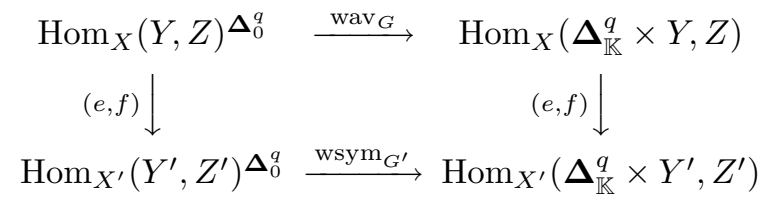

is commutative.

(4) If $q=0$, then $\operatorname{wav}_{G}$ is the identity map of $\operatorname{Hom}_{X}(Y, Z)$.

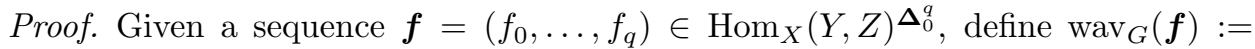
$f^{\prime} \in \operatorname{Hom}_{X}\left(\boldsymbol{\Delta}_{\mathbb{K}}^{q} \times Y, Z\right)$ to be the morphism such that

$$
\left(\operatorname{wsym}_{G}^{d(G)} \circ \mathrm{w}_{G}\right)\left(f_{0}, \ldots, f_{q}\right)=\left(f^{\prime}, \ldots, f^{\prime}\right)
$$

see Lemma 1.9. Properties (1)-(4) follow from the corresponding properties of $\mathrm{w}_{G}$ and $\operatorname{wsym}_{G}$.

Proof of Corollary 0.2. Take $X=Y:=$ Spec $\mathbb{K}$ in Theorem 1.11, and consider the $G$-torsor $\underline{Z}:=G$. Choose any base point $z \in Z$; this defines an isomorphism of left $G(\mathbb{K})$-sets $\underline{Z}(\mathbb{K}) \cong Z$. The weight sequence $\boldsymbol{w}$ can be considered as a $\mathbb{K}$-rational point of $\boldsymbol{\Delta}_{\mathbb{K}}^{q}$, and we define

$$
\operatorname{wav}_{G, \boldsymbol{w}}(\boldsymbol{z}):=\operatorname{wav}_{G}(\boldsymbol{z})(\boldsymbol{w}) \in Z .
$$

If we were to choose another base point $z^{\prime} \in Z$, this would amount to applying an automorphism of the torsor $\underline{Z}$, namely right multiplication by some element of $G(\mathbb{K})$. Due to the functoriality of $\operatorname{wav}_{G}$ the point $\operatorname{wav}_{G, \boldsymbol{w}}(\boldsymbol{z})$ will be unchanged.

The properties of this set-theoretical averaging process are now immediate consequences of the corresponding properties of the geometric average.

Remark 1.12. Z. Reichstein observed that our averaging process provides a new proof (in characteristic 0 ) of the fact that a unipotent group $G$ is special, namely any $G$-torsor $Z$ over $\mathbb{K}$ has a $\mathbb{K}$-rational point. Let us explain the idea.

Let $z_{0} \in Z$ be some closed point. Choose a finite Galois extension $L$ of $\mathbb{K}$ containing the residue field $\boldsymbol{k}\left(z_{0}\right)$. Let $\Gamma$ be the Galois group of $L$ over $\mathbb{K}$, which acts on the set $Z(L)$. Let $z_{0}, \ldots, z_{q} \in Z(L)$ be the $\Gamma$-conjugates of $z_{0}$. The group $\Gamma$ acts on the sequence $z:=\left(z_{0}, \ldots, z_{q}\right)$ by permutations. Thus the simultaneous action of $\Gamma$ on

$$
Z(L)^{\Delta_{0}^{q}}=\operatorname{Hom}_{\operatorname{Spec} \mathbb{K}}(\operatorname{Spec} L, Z)^{\boldsymbol{\Delta}_{0}^{q}}
$$

fixes $\boldsymbol{z}$

We know that the operator $\operatorname{wav}_{G}$ is symmetric. And functoriality says that the action of the Galois group on $\operatorname{Spec} L$ is also respected. Since $\boldsymbol{z}$ is fixed by the simultaneous action of $\Gamma$, so is $\operatorname{wav}_{G}(\boldsymbol{z})$. Take the uniform weight sequence $\boldsymbol{w}:=\left(\frac{1}{q+1}, \ldots, \frac{1}{q+1}\right)$ and define $z^{\prime}:=\operatorname{wav}_{G}(\boldsymbol{z})(\boldsymbol{w}) \in Z(L)$. Because $\boldsymbol{w}$ is fixed by the permutation group we conclude that $z^{\prime}$ is $\Gamma$-invariant, and hence $z^{\prime} \in Z(\mathbb{K})$.

Remark 1.13. Theorem 1.11 has a rather obvious parallel in differential geometry. Indeed, a simply connected nilpotent Lie group is the same as the group $G(\mathbb{R})$ of rational points of a unipotent algebraic group $G$ over $\mathbb{R}$. 


\section{Simplicial Sections}

In this section we show how the averaging process is used to obtain simplicial sections of certain bundles.

Suppose $H$ and $G$ are affine group schemes over $\mathbb{K}$, and $H$ acts on $G$ by automorphisms. Namely, there is a morphism of schemes $H \times G \rightarrow G$ which for every $\mathbb{K}$-scheme $Y$ induces a group homomorphism $H(Y) \rightarrow \operatorname{Aut}_{\text {Groups }}(G(Y))$. Then $H \times G$ has a structure of a group scheme, and we denote this group by $H \ltimes G$; it is a geometric semi-direct product.

Recall that an affine group scheme $G$ is called pro-unipotent if it is isomorphic to an inverse limit $\lim _{\leftarrow i} G_{i}$ of an inverse system $\left\{G_{i}\right\}_{i>0}$ of (finite type affine) unipotent groups. One may assume that each of the morphisms $G \rightarrow G_{i} \rightarrow G_{i-1}$ is surjective. Thus $G_{i} \cong G / N_{i}$ where $N_{i}$ is a normal closed subgroup of $G$.

We will be concerned with the following geometric situation.

Scenario 2.1. Let $H \ltimes G$ be an affine group scheme over $\mathbb{K}$. Assume $G$ is prounipotent and, moreover, there exists a sequence $\left\{N_{i}\right\}_{i \geq 0}$ of $H$-invariant closed normal subgroups of $G$ such that $G \cong \lim _{\leftarrow i} G / N_{i}$ and each $G / N_{i}$ is unipotent. Let $\pi: Z \rightarrow X$ be an $H \ltimes G$-torsor over $X$ which is locally trivial for the Zariski topology of $X$. Define $\bar{Z}:=Z / H$ and let $\bar{\pi}: \bar{Z} \rightarrow X$ be the projection.

Theorem 2.2. Assume Scenario 2.1. Suppose $U \subset X$ is an open set and $\sigma_{0}, \ldots, \sigma_{q}: U \rightarrow \bar{Z}$ are sections of $\bar{\pi}$. Then there exists a morphism

$$
\sigma: \boldsymbol{\Delta}_{\mathbb{K}}^{q} \times U \rightarrow \bar{Z}
$$

such that the diagram

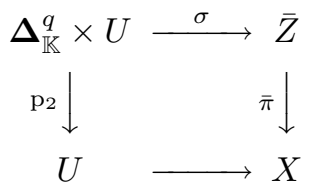

is commutative. The morphism $\sigma$ depends functorially on $U$ and simplicially on the sequence $\left(\sigma_{0}, \ldots, \sigma_{q}\right)$. If $q=0$, then $\sigma=\sigma_{0}$.

Proof. We might as well assume that $U=X$. Consider the quotient $Z / G$. Since $G$ is normal in $H \ltimes G$ it follows that $Z / G$ is a torsor under $H \times X$. We write $\pi_{H}: Z \rightarrow \bar{Z}=Z / H$ and $\pi_{G}: Z \rightarrow Z / G$ for the projections.

Pick an open set $V \subset X$ which trivializes $\pi: Z \rightarrow X$. We write $\left.Z\right|_{V}:=\pi^{-1}(V)$. Because $\left.\pi_{H}\right|_{V}:\left.\left.Z\right|_{V} \rightarrow \bar{Z}\right|_{V}$ is a trivial torsor under $H \times\left.\bar{Z}\right|_{V}$, we can lift the sections $\sigma_{0}, \ldots, \sigma_{q}$ to sections $\tilde{\sigma}_{0}, \ldots, \tilde{\sigma}_{q}: V \rightarrow Z$ such that $\pi_{H} \circ \tilde{\sigma}_{j}=\sigma_{j}$. Furthermore, since $\pi_{G}: Z \rightarrow Z / G$ is $H$-equivariant and $Z / G$ is a torsor under $H \times X$, it follows that we can choose $\tilde{\sigma}_{0}, \ldots, \tilde{\sigma}_{q}$ such that $\pi_{G} \circ \tilde{\sigma}_{j}=\tau$ for some section $\tau: V \rightarrow Z / G$.

Let $\left.F \subset Z\right|_{V}$ be the fiber over $\tau$, i.e., $F:=V \times_{Z / G} Z$ via the morphisms $\pi_{G}: Z \rightarrow Z / G$ and $\tau: V \rightarrow Z / G$. Then $F$ is a torsor under $G \times V$, and $\tilde{\sigma}_{0}, \ldots, \tilde{\sigma}_{q} \in$ 
$\operatorname{Hom}_{X}(V, F)$. See the diagram below.

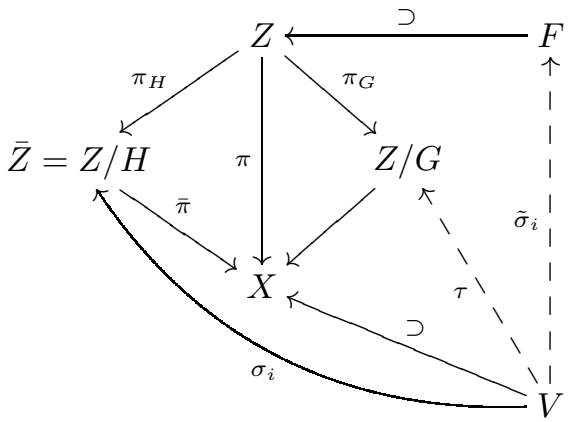

For any $i$ define $F_{i}:=F / N_{i}$, which is a torsor under $\left(G / N_{i}\right) \times V$. Let $\alpha_{i}: F \rightarrow F_{i}$ be the projection, so $\alpha_{i} \circ \tilde{\sigma}_{j} \in \operatorname{Hom}_{X}\left(V, F_{i}\right)$. By Theorem 1.11 we get an average

$$
\rho_{i}:=\operatorname{wav}_{G / N_{i}}\left(\alpha_{i} \circ \tilde{\sigma}_{0}, \ldots, \alpha_{i} \circ \tilde{\sigma}_{q}\right): \boldsymbol{\Delta}_{\mathbb{K}}^{q} \times V \rightarrow F_{i} .
$$

The functoriality of wav says that the $\rho_{i}$ form an inverse system, and we let

$$
\rho:=\lim _{\leftarrow i} \rho_{i}: \boldsymbol{\Delta}_{\mathbb{K}}^{q} \times V \rightarrow F
$$

and

$$
\sigma:=\pi_{H} \circ \rho: \Delta_{\mathbb{K}}^{q} \times V \rightarrow \bar{Z} .
$$

We claim that the morphism $\sigma$ does not depend on the choice of the section $\tau: V \rightarrow Z / G$. Suppose $\tau^{\prime}: V \rightarrow Z / G$ is another such section. Let $F^{\prime}$ be the fiber over $\tau^{\prime}$, and let $\rho^{\prime}: \boldsymbol{\Delta}_{\mathbb{K}}^{q} \times V \rightarrow F^{\prime}$ be the corresponding morphism as in (2.4). Now $\tau^{\prime}=h \cdot \tau$ for some morphism $h: V \rightarrow H$. Then $F^{\prime}=h \cdot F$, and $h: F \rightarrow F^{\prime}$ is a $G \times V$-equivariant morphism of torsors, with respect to the groupscheme automorphism $\operatorname{Ad}(h): G \times V \rightarrow G \times V$. The new lift of $\sigma_{j}$ is $\tilde{\sigma}_{j}^{\prime}:=h \cdot \tilde{\sigma}_{j}:$ $V \rightarrow F^{\prime}$. Define $F_{i}^{\prime}:=F^{\prime} / N_{i}$, and let $\rho_{i}^{\prime}: \Delta_{\mathbb{K}}^{q} \times V \rightarrow F_{i}^{\prime}$ be the morphism as in (2.3). Since $N_{i} \times V=\operatorname{Ad}(h)\left(N_{i} \times V\right)$, we get a group-scheme automorphism $\operatorname{Ad}(h):\left(G / N_{i}\right) \times V \rightarrow\left(G / N_{i}\right) \times V$, and a $\left(G / N_{i}\right) \times V$-equivariant morphism of torsors $h: F_{i} \rightarrow h \cdot F_{i}^{\prime}$. By functoriality of wav (property (3) in Theorem 1.11) it follows that $\rho_{i}^{\prime}=h \cdot \rho_{i}$. Therefore, $\rho^{\prime}=h \cdot \rho$, and $\pi_{H} \circ \rho^{\prime}=\pi_{H} \circ \rho=\sigma$.

Property (2) in Theorem 1.11implies that $\sigma$ depends simplicially on $\left(\sigma_{0}, \ldots, \sigma_{q}\right)$.

Finally, take an open covering $X=\bigcup V_{j}$ such that each $V_{j}$ trivializes $\pi: Z \rightarrow X$, and let $\sigma_{j}: \boldsymbol{\Delta}_{\mathbb{K}}^{q} \times\left. V_{j} \rightarrow \bar{Z}\right|_{V_{j}}$ be the morphism constructed in (2.5). Since no choices were made, we have $\left.\sigma_{j}\right|_{V_{j} \cap V_{k}}=\left.\sigma_{k}\right|_{V_{j} \cap V_{k}}$ for any two indices. Therefore, these sections can be glued to a morphism $\sigma: \boldsymbol{\Delta}_{\mathbb{K}}^{q} \times X \rightarrow \bar{Z}$. The functorial and simplicial properties of $\sigma$ are clear from its construction.

Let $X$ be a $\mathbb{K}$-scheme, and let $X=\bigcup_{i=0}^{m} U_{(i)}$ be an open covering, with inclusions $g_{(i)}: U_{(i)} \rightarrow X$. We denote this covering by $\boldsymbol{U}$. For any multi-index $\boldsymbol{i}=\left(i_{0}, \ldots, i_{q}\right) \in \boldsymbol{\Delta}_{q}^{m}$, we write $U_{\boldsymbol{i}}:=\bigcap_{j=0}^{q} U_{\left(i_{j}\right)}$, and we define the scheme $U_{q}:=\coprod_{\boldsymbol{i} \in \boldsymbol{\Delta}_{q}^{m}} U_{\boldsymbol{i}}$. Given $\alpha \in \boldsymbol{\Delta}_{p}^{q}$ and $\boldsymbol{i} \in \boldsymbol{\Delta}_{q}^{m}$, there is an inclusion of open sets $\alpha_{*}: U_{\boldsymbol{i}} \rightarrow U_{\alpha_{*}(\boldsymbol{i})}$. These patch to a morphism of schemes $\alpha_{*}: U_{q} \rightarrow U_{p}$, making $\left\{U_{q}\right\}_{q \in \mathbb{N}}$ into a simplicial scheme. The inclusions $g_{(i)}: U_{(i)} \rightarrow X$ induce inclusions $g_{i}: U_{i} \rightarrow X$ and morphisms $g_{q}: U_{q} \rightarrow X$; and one has the relations $g_{p} \circ \alpha_{*}=g_{q}$ for any $\alpha \in \Delta_{p}^{q}$. 
Definition 2.6. Let $\pi: Z \rightarrow X$ be a morphism of $\mathbb{K}$-schemes. A simplicial section of $\pi$ based on the covering $\boldsymbol{U}$ is a sequence of morphisms

$$
\boldsymbol{\sigma}=\left\{\sigma_{q}: \boldsymbol{\Delta}_{\mathbb{K}}^{q} \times U_{q} \rightarrow Z\right\}_{q \in \mathbb{N}}
$$

satisfying the following conditions.

(i) For any $q$, the diagram

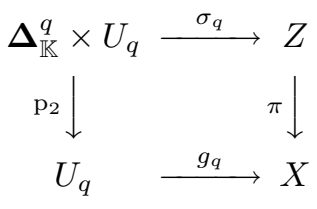

is commutative.

(ii) For any $\alpha \in \boldsymbol{\Delta}_{p}^{q}$, the diagram

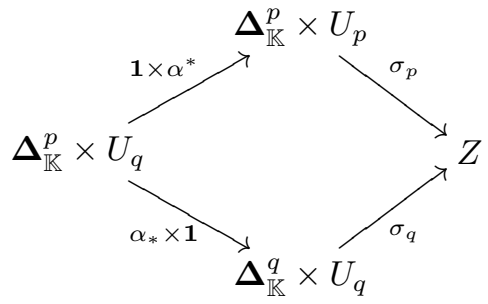

is commutative.
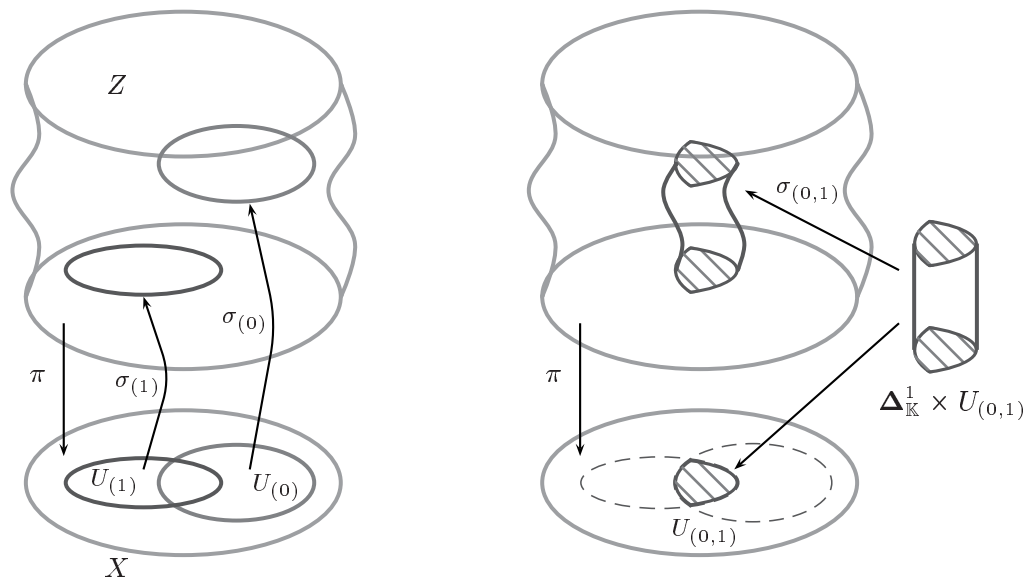

FiguRe 1. Simplicial sections, $q=1$. We start with sections over two open sets $U_{(0)}$ and $U_{(1)}$ in the left diagram; and we pass to a simplicial section $\sigma_{(0,1)}$ on the right. As can be seen, $\sigma_{(0,1)}$ interpolates between $\sigma_{(0)}$ and $\sigma_{(1)}$. 
Corollary 2.7. Assume Scenario 2.1, Let $\boldsymbol{U}=\left\{U_{(i)}\right\}_{i=0}^{m}$ be an open covering of $X$. Suppose that for any $i \in\{0, \ldots, m\}$ we are given some section $\sigma_{(i)}: U_{(i)} \rightarrow \bar{Z}$ of $\bar{\pi}$. Then there exists a simplicial section

$$
\boldsymbol{\sigma}=\left\{\sigma_{q}: \boldsymbol{\Delta}_{\mathbb{K}}^{q} \times U_{q} \rightarrow \bar{Z}\right\}_{q \in \mathbb{N}}
$$

based on $\boldsymbol{U}$, such that $\left.\sigma_{0}\right|_{U_{(i)}}=\sigma_{(i)}$ for all $i \in\{0, \ldots, m\}$.

Proof. For any multi-index $\boldsymbol{i}=\left(i_{0}, \ldots, i_{q}\right)$, we have sections $\sigma_{\left(i_{0}\right)}, \ldots, \sigma_{\left(i_{q}\right)}: U_{\boldsymbol{i}} \rightarrow$ $\bar{Z}$. Let $\sigma_{\boldsymbol{i}}: \boldsymbol{\Delta}_{\mathbb{K}}^{q} \times U_{\boldsymbol{i}} \rightarrow \bar{Z}$ be the morphism provided by Theorem 2.2, For fixed $q$ these patch to a morphism $\sigma_{q}: \boldsymbol{\Delta}_{\mathbb{K}}^{q} \times U_{q} \rightarrow \bar{Z}$. The functorial and simplicial properties in Theorem 2.2 imply that this is a simplicial section.

This result (with $H$ trivial) is illustrated in Figure 1

\section{REFERENCES}

[Bo] R. Bott, "Lectures on Characteristic Classes and Polarizations", Lecture Notes in Math. 279, Springer, Berlin, 1972. MR0362335 (50:14777)

[GK] I.M. Gelfand and D.A. Kazhdan, Some problems of differential geometry and the calculation of cohomologies of Lie algebras of vector fields, Soviet Math. Dokl. 12 (1971), no. 5, 1367-1370.

[Ho] G. Hochschild, "Basic Theory of Algebraic Groups and Lie Algebras," Springer-Verlag, 1981. MR0620024 (82i:20002)

[HY] R. Hübl and A. Yekutieli, Adelic Chern forms and applications, Amer. J. Math. 121 (1999), 797-839. MR1704478 (2000h:14016)

[Ko] M. Kontsevich, Deformation quantization of Poisson manifolds, Lett. Math. Phys. 66 (2003), no. 3, 157-216. MR 2062626 (2005i:53122)

[Ye] A. Yekutieli, Deformation Quantization in Algebraic Geometry, Adv. Math. 198 (2005), 383-432. MR 2183259

Department of Mathematics, Ben Gurion University, Be’er Sheva 84105, Israel

E-mail address: amyekut@math.bgu.ac.il 